\section{ONOMAREDIN}

Revista semestral de lingüística, filología y traducción
PONTIFICIA UNIVERSIDAD CATÓLICA DE CHILE CATOLICA DE CHILE
FACULTAD DE LETRAS

\title{
Desverbalización y estructuras sintácticas en interpretación simultánea
}

Deverbalization and gramatical structure in simultaneous interpreting

\section{Ginette Gabriela Castro Yáñez \\ Universidad Católica de Temuco Chile}

Waldo Boris Chaparro Inzunza

Universidad de Concepción Chile

Ginette Castro: Departamento de Lenguas y Traducción, Facultad de Artes y Humanidades, Universidad Católica de Temuco. Chile. Correo electrónico: gcastro@uct.cl

Waldo Chaparro: Programa de Magíster en Lingüística Aplicada, Facultad de Humanidades y Arte, Universidad de Concepción. Chile. Correo electrónico: waldochaparro@udec.cl 


\section{Resumen}

Se han propuesto diferentes modelos cognitivos y psicolingüísticos con la finalidad de explicar y describir las características de la interpretación simultánea. Uno de ellos es el del procesamiento basado en el sentido, en el que se enmarca el proceso de desverbalización, consistente en la extracción del significado del texto de su estructura gramatical. En este estudio exploratorio y transversal de carácter cualitativo se busca obtener evidencia de este proceso por medio de la comparación de las estructuras gramaticales que presentan el texto origen y meta. Para ello se analizó las características estructurales del output de seis participantes, a partir de lo que se detectó diez fenómenos, cuyas características se describen y que presentan evidencias empíricas a favor y en contra del proceso de desverbalización.

Palabras clave: interpretación simultánea; psicolingüística; desverbalización; procesamiento en base al sentido; sintaxis.

\section{Abstract}

Different cognitive and psycholinguistic models have been proposed in order to explain and describe the characteristics of simultaneous interpreting. One of those models is the meaningbased processing, in which the deverbalization process is found. It consists in the extraction of the text's meaning out of its grammatical structure. This transversal, qualitative and exploratory research aims to obtain evidence about this process, through the comparison of the grammatical structures presented by both the source and target texts. To achieve this goal, the structural features from six participants' output were analyzed. Ten phenomena were detected and their characteristics were described. They present empiric evidence for and against deverbalization.

Keywords: simultaneous interpretation; psycholinguistics; deverbalization; meaning-based processing; syntax. 


\section{Introducción}

Dentro de las variantes de la actividad interpretativa se encuentra la modalidad simultánea, que es aquella en la que el intérprete escucha y habla al mismo tiempo que el orador (De Groot, 1997). Los profesionales dedicados a esta actividad escuchan las oraciones pronunciadas en una lengua y, unos segundos después, producen nuevas oraciones en un idioma diferente, conservando el sentido (Isham, 1994). Así, la audiencia puede oír el traspaso del discurso a otra lengua a medida que es pronunciado (Paneth, 1957). Este tipo de interpretación representa la más prototípica en la actualidad (Kutz, 2010) y debería ser estudiada porque se cuenta con poca evidencia empírica sobre los procesos involucrados en ella. Entre los diferentes campos que se entrecruzan al momento de investigar la interpretación simultánea, se encuentra la psicolingüística, disciplina a partir de la cual numerosos autores han intentado entregar respuestas a las muchas interrogantes que genera la actividad translativa oral (Kirchhoff, 1976; Isham, 1994; Moser-Mercer, 1997; Kutz 2010).

Se ha propuesto diferentes teorías y modelos de procesos que han descrito qué ocurre durante la tarea interpretativa, entre los que encontramos el procesamiento en base al sentido y el proceso de desverbalización. Este último consiste en la extracción del sentido comunicativo del mensaje en lengua origen y el descarte de la forma lingüística del texto, por lo que, según esta teoría, el intérprete utilizaría sólo la información no verbal extraída para producir el mensaje nuevo en lengua meta (Isham, 1994; Moser-Mercer, 1997; Dam, 1998; Gile, 2000; Kutz, 2010).

En las últimas décadas se han realizado diversas revisiones bibliográficas y se han publicado varios documentos sobre este tema (Kirchhoff, 1976; Isham, 1994; Gile, 2000; Pöchhacker y Shlesinger, 2002; Kutz, 2010; Seeber y Kerzel, 2011). Sin embargo, se ha señalado que no existe certeza empírica de este fenómeno ni si es más bien prescriptivo o descriptivo (Isham, 1994; Gile, 2003). Por lo tanto, se considera de interés para esta área realizar un estudio empírico que use el proceso de desverbalización como fundamento.

\subsection{La interpretación como proceso}

La interpretación es una actividad comunicativa manifestada de manera lingüística y paralingüística y a la vez es una actividad cognitivo-psicológica (Kutz, 2010). El carácter psicocognitivo en la definición anterior está dado por los procesos mentales complejos que involucra el traspaso de un discurso en una lengua origen a otro en lengua meta (en esta investigación se utilizarán los términos texto fuente, discurso origen, discurso fuente e input para hacer referencia al discurso pronunciado por el orador; para el discurso pronunciado por el intérprete en lengua meta, se usará los términos texto meta, discurso meta y output).

Entre las fases del procesamiento del texto a nivel cognitivo en la interpretación se encuentra el proceso de desverbalización, que surgió en la denominada Escuela de París y que está estrechamente relacionado con la theorie du sens (teoría del sentido), ampliamente difundida en el ámbito de la traducción (Kutz, 2010). Donde sea que se discuta el estudio de la interpretación desde una perspectiva más amplia e histórica, se hace necesariamente referencia al concepto de desverbalización y a la theorie du sens, concebida y formulada por Seleskovitch (Pöchhacker y Shlesinger, 2002).

Kutz (2010) explica el modelo interpretativo basado en el sentido resumiéndolo en tres fases. La primera es una fase verbal que involucra la percepción auditiva, la recepción y la comprensión del discurso. Luego se produce una fase no verbal, que es el procesamiento mismo del texto, en la cual el intérprete almacena el sentido. Finalmente ocurre una fase verbal, que es la reproducción (o expresión) del sentido en la lengua meta. Esto significa que el discurso en lengua 
origen es procesado de manera tal que se desverbaliza transformándose en un mensaje completamente independiente del lenguaje para luego ser recodificado en la lengua meta. Es decir, en la memoria del intérprete no queda ningún rastro de la forma lingüística del texto origen antes de la reproducción del output (Gile, 2000).

Según esta misma teoría, no existe recuperación del verbatim, puesto que sólo se ha guardado el contenido y no la forma del texto (MoserMercer, 1997). Posteriormente se construye un discurso en la lengua meta en base al sentido extraído y retenido por el intérprete y de acuerdo a las reglas gramaticales de este último idioma (Isham, 1994). De acuerdo con Dam (1998), el texto interpretado se produce principalmente siguiendo una representación no verbal del significado del texto origen y sólo de manera excepcional en base a su forma lingüística.

Según los modelos de Kirchhoff (1976) y Kitano (1994, en Kutz, 2010), la interpretación simultánea es una actividad de carácter secuencial e incremental que termina con la producción del texto meta y en la que, al igual que en la descripción de Kutz (2010), la comprensión es de gran importancia como fase inicial. En este sentido, Seeber (2011) señala que el intérprete generalmente necesita oír el discurso en la lengua origen y comprenderlo antes de proceder a codificarlo y producir el discurso en la lengua meta. Se requiere entonces de una comprensión cabal del input como etapa previa a su desverbalización.

\subsection{Calco estructural e interpretación simultánea}

Considerando lo que se ha descrito acerca del proceso desverbalizador, podría establecerse una relación entre ese fenómeno y el calco morfosintáctico en la actividad interpretativa simultánea.

El calco se define como una construcción imitativa presente en una oración, cuyos componentes toman un orden ajeno a la lengua propia e imitan el de una lengua extranjera (García, citado en Reyes, 2006). Esta definición se complementa por lo señalado por Francesconi (2004), para quien un calco es una traducción directa y consiste en la imitación del esquema formal de la palabra o del orden sintáctico (estructural) del texto original, definición muy cercana a la propuesta por Gerding (2008). Por ejemplo, un calco estructural entre el español y el inglés, sobre todo en el fenómeno del spanglish, es la utilización de palabras españolas para construir una oración en base al orden de la oración inglesa: Sé cómo bailar (I know how to dance), en lugar de sé bailar (Marcos-Marín, 2005).

Se podría suponer, por lo tanto, que se encontraría poca o ninguna similitud entre la estructura de las oraciones del discurso origen y la estructura de las del discurso meta. Es decir, si la desverbalización ocurre durante el procesamiento del texto, y es tal como se ha descrito, no debería haber calco morfosintáctico ni similitud de la forma de las oraciones o estos fenómenos deberían ser muy escasos.

\section{Método}

Según lo expuesto en los párrafos anteriores y en consideración al fenómeno de la desverbalización, el objetivo del presente estudio consistió en obtener evidencia de este proceso por medio del análisis y observación de calcos sintácticos en el output de una interpretación simultánea. En concordancia, la hipótesis plantea que la presencia o ausencia de calco sintáctico en el output en la interpretación simultánea debería permitir la obtención de evidencia acerca del proceso de desverbalización. Esta evidencia debería refutar lo que se ha señalado en la literatura acerca del proceso cuando se detecte calco de estructuras o reafirmar aquello cuando no se presente calco estructural.

\subsection{Participantes}

Participaron de manera voluntaria seis intérpretes egresados de la carrera de Traducción 
e Interpretación en idiomas extranjeros de la Universidad de Concepción, todos ellos de características similares, esto es, español como lengua A y alemán como lengua B o C. De acuerdo a Kutz (2010), la lengua A corresponde a la lengua materna, la B a la lengua extranjera activa (de mayor competencia), mientras que la lengua C es la lengua extranjera pasiva (de menor competencia). Previamente a la toma de muestras realizada en un laboratorio de interpretación de la Universidad de Concepción, se informó a los participantes acerca del tema del discurso que deberían interpretar para que pudieran documentarse. El número reducido de participantes se debe a la naturaleza psicolingüística de esta investigación, disciplina en la que es común analizar un fenómeno de manera acotada en pocos sujetos o incluso se realizan estudios de caso. Por lo general, tampoco se puede encontrar intérpretes dispuestos a participar en este tipo de investigaciones (Gile, 2000).

\subsection{Materiales}

Se utilizó un discurso de la canciller alemana Angela Merkel disponible en línea. El discurso consta de 1.161 palabras y se redujo a 517 palabras, extensión similar a la de textos utilizados en otras investigaciones de este tipo (Gerver, 1969). El texto lo leyó y grabó un hablante nativo de lengua alemana, intérprete profesional y docente de la Universidad de Concepción. La velocidad del input fue de 112,8 palabras/minuto, que es considerada óptima, ya que una velocidad mayor (por ejemplo: 150 palabras/minuto) afecta la calidad de la interpretación (Kutz, 2010).

\section{Resultados}

El análisis comparativo de los textos en alemán y español permitió la detección, en el texto meta, de diez fenómenos de diferenciación o semejanza de la forma de las oraciones de ambas versiones del mismo texto (véase la tabla 1 ).

A continuación se define y ejemplifica cada fenómeno. En los ejemplos se subraya el fragmento afectado.

\section{TABLA 1}

Fenómenos detectados y cantidad de ocurrencias

\begin{tabular}{c|c} 
Fenómenos & Ocurrencias \\
\hline Compresión & 17 \\
\hline Adición de un elemento nuevo & 11 \\
\hline Adición implícita en el texto origen & 33 \\
\hline Unión de oraciones & 29 \\
\hline Omisión sin compromiso de sentido & 77 \\
\hline Omisión con compromiso de sentido & 40 \\
\hline Error & 50 \\
\hline Inversión de elementos & 14 \\
\hline Orden similar de elementos & 36
\end{tabular}

\subsection{Compresión}

Se produce cuando segmentos del texto origen se presentan de manera más comprimida y resumida en el texto meta.

\section{Ejemplo:}

Ich möchte hier gleich zu Beginn noch wiederholen: Afghanistan hat die Unterstützung...

[Desde el comienzo, quisiera decirlo nuevamente: Afganistán cuenta con el apoyo...]

Debo repetirlo: Afganistán tiene el apoyo...

Podría señalarse que se desverbaliza el discurso fuente y luego se reverbaliza en base a su sentido, pero con una estructura oracional que difiere en longitud a la del texto en lengua origen.

\subsection{Expansión}

Se describe como la utilización de mayor cantidad de constituyentes sintácticos en lengua meta para expresar un segmento de menor extensión en lengua origen.

\section{Ejemplo:}

In diesem Sinne wünsche ich Ihnen, Herr Präsident, und der gesamten Konferenz viel Erfolg. 
[En este sentido, le deseo a usted, señor Presidente, y a toda la conferencia, mucho éxito.]

En este sentido, señor Presidente y a todos ustedes miembros y participantes de esta conferencia, les deseo mucho éxito.

Se observa que en los fragmentos expandidos se trabaja sobre la base del sentido del texto y no según su forma, ya que el sentido se mantiene, pero la forma se modifica en relación con una mayor longitud y un número mayor de constituyentes

\subsection{Adición de un elemento nuevo}

Corresponde a la aparición de una palabra o frase en el texto meta cuya presencia no se advierte en el texto origen.

Ejemplo:

Afghanistan kann auf unsere Unterstützung zählen, und zwar nicht nur die Regierung und die Institutionen..

[Afganistán puede contar con nuestro apoyo; y no tan sólo el gobierno y las instituciones...]

Afganistán puede contar con nuestra ayuda, y no tan sólo los nuevos gobiernos o instituciones...

Se genera un cambio evidente de la forma del texto, al añadirse un nuevo elemento. Sin embargo, tal cambio afecta también el sentido a transmitir.

\subsection{Adición implícita en el texto origen}

Corresponde a una explicitación o expresión clara y visible de un elemento del texto meta que se encuentra contenido en el sentido y no en la forma del texto origen.

Ejemplo:

Dann allerdings wird unsere Arbeit nicht beendet sein. Dann wird es darum gehen..

[Sin embargo, nuestro trabajo no habrá concluido. Entonces se tratará...]

Sin embargo, nuestro trabajo no habrá concluido, ya que se tratará..

Según se observa en el ejemplo, este fenómeno da indicios de una desverbalización integral y una comprensión cabal del texto origen.

\subsection{Unión de oraciones}

Consiste en la aparición de una sola oración en un fragmento del texto meta que, en el texto origen, está conformado por dos o más oraciones.

Ejemplo:

Aber wir müssen auch sagen: // Es lohnt sich, auch zehn Jahre später...

[Sin embargo, también debemos decir: Vale la pena, incluso después de diez años...]

Sin embargo, también debemos decir que vale la pena, después de diez años..

Como se aprecia en el ejemplo, es notorio el cambio en la estructura del texto origen, asociado además a un correcto traspaso del sentido.

\subsection{Omisión sin compromiso de sentido}

Entendemos por omisión sin compromiso de sentido al fenómeno en el que un elemento o fragmento del texto fuente no aparece interpretado en el texto meta.

\section{Ejemplo:}

Meine Damen und Herren, die afghanische Regierung und das afghanische Volk stehen zweifellos vor großen Herausforderungen.

[Damas y caballeros, no cabe duda de que el gobierno y el pueblo afgano se enfrentan a grandes desafíos.]

El gobierno afgano y el pueblo afgano enfrentan grandes desafíos, sin duda.

En este caso, tal elisión, que constituye una modificación en la superficie del texto, no provoca cambio ni alteración del sentido.

\subsection{Omisión con compromiso de sentido}

Corresponde a la no aparición de elementos con una mayor carga semántica del texto origen en el texto meta, lo que provoca un cambio, la pérdida o la entrega errónea de sentido.

Ejemplo:

Es ist nicht ganz einfach...

[No es tan fácil...]

Es fácil... 
Como se observa, el cambio en la forma del texto, representado por la elisión de elementos como nicht [no], afecta también al sentido.

\subsection{Error}

Se consideran en el presente trabajo, ya que involucran cambios tanto de sentido como de forma del fragmento del texto que afectan.

Ejemplo:

... trafen sich in Deutschland, hier in Bonn, Delegationen aus aller Welt unter der Ägide der Vereinten Nationen..

[... se reunieron en Alemania, aquí en Bonn, delegados de todo el mundo con el patrocinio de las Naciones Unidas...]

.. se han reunido en Bonn delegaciones de todo el mundo y con delegados de las Naciones Unidas.

Se observa la entrega de información falsa, lo que permite detectar un procesamiento defectuoso del texto, ya que el sentido no se mantiene al final del proceso interpretativo.

\subsection{Inversión de elementos}

En el texto origen se observan elementos que siguen un orden lineal o conforman una secuencia con una configuración determinada.

Ejemplo:

Von hoher Bedeutung ist auch der politische Prozess.

[De gran importancia es también el proceso político.]

El proceso político es de gran importancia.

En este caso, los constituyentes de la oración aparecen con un orden diferente en el texto meta; sin embargo, no se producen alteraciones en el sentido.

\subsection{Orden similar de elementos}

Se encuentra en los casos en que los elementos de una oración o de un fragmento del texto origen, que tienen un orden determinado, se configuran de manera similar en el mismo fragmento u oración del texto meta.
Ejemplo:

\begin{tabular}{|c|c|c|c|c|c|c|}
\hline \multicolumn{3}{|c|}{ In diesem Sinne } & \multicolumn{3}{|c|}{ wünsche ich Ihnen, } & Herr \\
\hline \multicolumn{3}{|c|}{ Es por eso } & \multicolumn{3}{|c|}{ que le deseo, } & señor \\
\hline Präsident, & und & der & Gesamten & Konferenz & viel & Erfolg. \\
\hline Presidente, & $y$ & $\mathrm{a}$ & toda la & conferencia & mucho & éxito. \\
\hline
\end{tabular}

[En este sentido, le deseo, señor presidente, y a toda la conferencia, mucho éxito.]

En el caso de este fenómeno, se evidencia un correcto traspaso del sentido asociado a la misma estructura del texto origen.

\section{Discusión}

Como se ha presentado en el apartado anterior, algunos de los fenómenos detectados sirven como apoyo a lo descrito en la literatura revisada acerca del proceso de desverbalización (Isham, 1994; Moser-Mercer, 1997; Gile, 2000; Kutz, 2010), ya que en ellos se producen cambios en la estructura de las oraciones del texto meta y se mantiene el sentido, que aparece sin modificaciones al final del proceso interpretativo. Estos fenómenos son la compresión, expansión, adición implícita en el texto origen, unión de oraciones, omisión sin compromiso de sentido e inversión de elementos. Sus características permiten señalar que se trabajaría sólo con la información extraída descartándose la forma del texto, lo que apoya la manera en que Gile (1990, en Isham, 1994) explica el proceso de desverbalización.

Además, el análisis de los fenómenos detectados en la superficie del texto permite señalar que la desverbalización sería un proceso total, según el cual el texto sería procesado sólo en relación con su sentido, lo que respondería algunas de las interrogantes planteadas por Gile (2003) acerca de la parcialidad o totalidad del proceso. Asimismo es importante mencionar que, en todos estos fenómenos, no se producen errores gramaticales de forma ni de sentido, lo que indicaría que, al producirse la desverbaliza- 
ción, se extrae la información y el intérprete produce el texto en lengua meta según sus reglas gramaticales, lo que concuerda con lo que Isham (1994) señala.

No obstante lo anterior, diferente es la situación de los fenómenos de adición de un elemento nuevo, omisión con compromiso de sentido y error. En ellos, se evidenciaría que la desverbalización no se produce de la manera descrita y propuesta por los autores ya nombrados. Sin embargo, su ocurrencia podría estar relacionada con otros procesos previos o posteriores a la desverbalización, como lo son la comprensión del input y la producción del output, que tienen un rol importante en la actividad interpretativa y están relacionados directamente con la desverbalización (Baddeley, 1999; Kutz, 2010).

Sobre los fenómenos error y omisiones con compromiso de sentido, especialmente cuando estas últimas afectan fragmentos completos del texto, se podría señalar que, al producirse mermas en la comprensión, no se puede realizar una interpretación exitosa, ya que la desverbalización se basa en este primer subproceso. Así, la interpretación no se produce de manera óptima, lo que apoya la importancia que reviste el proceso de la comprensión del discurso original, tema tratado por algunos autores como Kirchhoff (1976) y Kitano (1994, en Kutz, 2010).

Algunos de los fenómenos también dan cuenta de una separación incompleta entre la forma del texto y el sentido, ya que no sólo se descarta la forma del texto, sino que también se pierde parte de la información. Un ejemplo de ello lo constituyen las omisiones con compromiso de sentido y algunos errores, lo que aporta evidencias de que en algunos segmentos del texto no se produciría la separación total a la que se refieren autores como Isham (1994) y Gile (2000). Podría ser entonces que, en estos fenómenos, no se realiza una desverbalización propiamente tal o que los subprocesos aledaños se ven afectados por algún motivo. Sin embargo, debido al carácter y las limitaciones del presente trabajo no es posible determinar de manera clara si el fenómeno se debió a lo anterior.

Por otra parte, la ocurrencia del fenómeno de orden similar de elementos morfosintácticos permite señalar que hay ocasiones en que la desverbalización no ocurriría en absoluto como se señala en la literatura, que es contrario a lo esperado. Esto mismo refuta la descripción que Isham (1994), Moser-Mercer (1997), Gile (2000) y Kutz (2010) postulan sobre la desverbalización.

Esta similitud de estructuras y la mantención del sentido indican la inexistencia de la etapa en que se extrae la información y posteriormente se descarta la forma del texto origen, lo que podría deberse a que, en esos fragmentos simplemente no se desverbalizó o no se produjo una separación mayor, debido a la vinculación de los segmentos de la lengua origen y de la lengua meta a la que Kirchhoff (1976) hace referencia. Esta vinculación está representada por patrones convencionales, frases estereotípicas y secuencias conocidas que en interpretación simultánea se relacionan sin dificultad.

El hecho de que en algunos casos se produzcan algunos fenómenos y otros no y que en ciertos fragmentos del texto algunos participantes hayan desverbalizado y en otros no, da indicios de la parcialidad de este proceso y se condice con los resultados de la investigación de Isham (1994). Sin embargo, en el presente trabajo es difícil determinar por qué se produjo esta situación. No obstante, hubo menos fenómenos que refutan la desverbalización, lo que afirma la idea expresada por Dam (1998), según la cual el texto interpretado se produce principalmente en base a una representación esencialmente no verbal del significado del texto origen y sólo de manera excepcional en base a la forma lingüística.

\section{Conclusión}

La determinación de distintos niveles de semejanza morfosintáctica permitió, en la investigación presentada, obtener evidencia de carácter empírico acerca del proceso de desver- 
balización y la manera en que este ha sido descrito y propuesto en la literatura especializada.

La detección de los fenómenos presentados determinó que el procesamiento del texto en interpretación simultánea es una tarea compleja que presenta retos no menores a la hora de su descripción, debido a la invisibilidad de los subprocesos involucrados y a que sólo se cuenta con datos de superficie. Sin embargo, se evidenció que el procesamiento del texto no es una actividad única, sino que involucra otros procesos como lo son la desverbalización, la comprensión y la producción del texto meta.

En relación con lo anterior, lo observado deja entrever que en algún punto del proceso cognitivo que constituye la interpretación simultánea, el cual parte con la percepción y comprensión del discurso origen y finaliza con la producción del discurso meta, ocurre la desverbalización. Como se evidenció en los resultados obtenidos, su aparición se ve afectada en ocasiones, lo que podría deberse a fenómenos a nivel de los otros subprocesos mencionados.

El trabajar sólo con los elementos relacionados con la forma sintáctica de las oraciones en las lenguas fuente y meta sirvió como una herramienta útil al momento de comprobar la existencia de la desverbalización. Sin embargo, se debe tener en cuenta que este estudio tiene limitaciones que son comunes a investigaciones de la misma naturaleza. En este caso los resultados se deben generalizar de manera muy cuidadosa y deben ser entendidos en el marco de la complejidad ecológica de una experiencia investigativa en interpretación.

Los resultados y las nuevas interrogantes que se abren a partir de ellos son de interés por la obtención de evidencia empírica en un campo en el que no existe suficiente información de este tipo. Consideramos entonces que el presente trabajo constituye un aporte tanto para los estudios en translatología emergentes en el país como para la disciplina en general.

\section{Bibliografía citada}

Baddeley, Alan, 1999: La memoria humana: teoría y praxis, Madrid: McGraw Hill.

DAm, Helle, 1998: "Lexical Similarity vs Lexical Dissimilarity in Consecutive Interpreting: A Product Oriented Study of Form Based vs Meaning Based Intepreting" en Franz PöchHACKER y Miriam SHLESINGER (eds.): The Interpreting Studies Reader, Londres y Nueva York: Routledge, 267-277.

De Groot, Annette, 1997: "The Cognitive Study of Translation and Interpretation: Three Approaches" en Joseph Danks, Gregory Shreve, Stephen Fountain y Michael McBeath (eds.): Cognitive Processes in Translation and Interpreting, Estados Unidos de Norteamérica: Sage Publications Inc, 25-56.

Francesconi, Armando, 2004: “¿Qué traducción? LoS métodos de traducción en el análisis contemporáneo" [http://www.ucm.es/info/especulo/nu mero27/traducc.html, fecha de consulta: 12 de enero de 2012].

Gerver, David, 1969: "The effects of source language presentation rate on the performance of simultaneous conference interpreters" en Franz PöchHACKer y Miriam SHLesinger (eds.): The Interpreting Studies Reader, Londres y Nueva York: Routledge, 52-67.

Gerding, Constanza, 2008: Traducción. Terminología de la Traducción, Concepción: Dirección de Docencia Universidad de Concepción.

GILE, Daniel, 2000: "Issues in Interdisciplinary Research into Conference Interpreting" en Birgitta Dimitrova y Kenneth Hyltenstam (eds.): Language Processing and Simultaneous Interpreting, Amsterdam, Philadelphia: John Benjamins Publishing Company, 89-106.

GILE, Daniel, 2003: "Justifying the deverbalization approach in the interpreting and translation classroom" [http://www.google.cl/url?sa=t\&rct $=j \& q=\&$ es $r c=s \&$ source $=$ web \&cd $=1 \& c a d=r j a \& v e d$ =0CDEQFjAA\&url=http\%3A\%2F\%2Fcirinandgile. 
com\%2F2003deverbtxt.doc\&ei=kTr-UPTZO5K49 gSchICoBw\&usg=AFQjCNEoKsiSMpeJtStQdxoTt hbD4CUjFw\&bvm=bv.41248874,d.eWU, fecha de consulta: 23 de octubre de 2011].

Gobierno Federal de Alemania, 2011: Rede von Bundeskanzlerin Angela Merkel anlässlich der Eröffnung der Internationalen Afghanistan-Konferenz [http://www.bundesregierung.de/Content/ DE/Rede/2011/12/2011-12-05-merkel-afgh-konf. html, fecha de consulta: 2 de febrero de 2012].

IsHAm, William, 1994: "Memory For Sentence Form After Simultaneous Interpretation: Evidence Both For And Against Deverbalization" en Sylvie Lambert y Barbara Moser-Mercer (eds.): Bridging the Gap: Empirical research in simultaneous interpretation, Amsterdam, Philadelphia: John Benjamins Publishing Company, 191-211.

KIRChHOFF, Hella, 1976: "Simultaneous Interpreting: Interdependence of variables in the interpreting process, interpreting models and interpreting strategies" en Franz PöchHacker y Miriam SHLESINGER (eds.): The Interpreting Studies Reader, Londres y Nueva York: Routledge, 110-119.

Kutz, Wladimir, 2010: Dolmetschkompetenz: Was muss der Dolmetscher wissen und können, Alemania: Europäischer Universitätsverlag.

Marcos-Marin, Francisco, 2005: "Pluralidad del español en los Estados Unidos de Norteamérica” [http://cvc.cervantes.es/lengua/anuario/anua rio_05/default.htm, fecha de consulta: 22 de enero de 2012].

Moser-Mercer, Barbara, 1997: "Beyond Curiosity: Can Interpreting Research Meet the Challenge?" en Joseph Danks, Gregory Shreve, Stephen Fountain y Michael Mcbeatr (eds.): Cognitive Processes in Translation and Interpreting, Estados Unidos de Norteamérica: Sage Publications Inc, 176-195.

Paneth, Eva, 1957: "An Investigation into Conference Interpreting" en Franz PöchHACKER y Miriam SHLESINGER (eds.): The Interpreting Studies Reader, Londres y Nueva York: Routledge, 30-41.
PöchHACker, Franz y Miriam Shlesinger (eds.), 2002: The Interpreting Studies Reader, Londres y Nueva York: Routlegde.

Reyes, Fabiola, 2006: "Interferencia léxica y sintáctica de la segunda lengua en la lengua materna en un seminario de iniciación a la traducción" [http://www.saber.ula.ve/bitstream/123456789/17261/2/articulo4.pdf, fecha de consulta: 24 de enero de 2012].

Seeber, Killian, 2011: "Cognitive load in simultaneous interpreting: Existing theories - new models" en Interpreting: International Journal of Research and Practice in Interpreting 13:2, John Benjamins Publishing Company, 176-204.

Seeber, Killian y Dirk Kerzel, 2011: "Cognitive Load in Simultaneous Interpreting: Model Meets Data" [http://www.unige.ch/fapse/PSY/persons/ kerzel/reprints/IJB.2012.pdf, fecha de consulta: 6 de enero de 2012]. 